\title{
Double Chamber Left Ventricle Associated With Severe Form of the Hypertrophic Cardiomyopathy and High Left Intracavitary Pressure
}

\author{
${\text { Ramush Bejiqi }{ }^{{ }^{\star}}, \text { Ragip Retkoceri }^{1}, \text { Hana Bejiqi }^{2}, \text { Naim Zeka }^{1}, \text { Arlinda Maloku }^{1}, \text { Majlinda Berisha }}^{1}$ \\ ${ }^{1}$ Division of Cardiology, Pediatric Clinic, University Clinical Center of Kosovo, Prishtina, Republic of Kosovo; ${ }^{2}$ Main Center of \\ Family Medicine, Prishtina, Republic of Kosovo
}

\author{
Citation: Bejiqi R, Retkoceri R, Bejiqi $\mathrm{H}$, Zeka \\ $\mathrm{N}$, Maloku A, Berisha M. Double Chamber Left \\ Ventricle Associated With Severe Form of the \\ Hypertrophic Cardiomyopathy and High Left \\ Intracavitary Pressure. Maced J Med Sci. 2013 \\ Sep 15; 6(3):281-284. http://dx.doi.org/10.3889/ \\ MJMS.1857-5773.2013.0315. \\ Key words: Double chamber left ventricle; \\ family screening; hypertrophic cardiomyopathy; \\ sudden death; echocardiography. \\ "Correspondence: Prof. Ramush Alil Bejiqi. \\ University Clinical Center of Kosovo, Pediatric \\ Clinic, Prishtina, Kosovo. Phone: oo377 44120 \\ 129. E-Mail: rbejiqi@hotmail.com \\ Received: 24-Mar-2013; Revised: 20-Aug- \\ 2013; Accepted: 01-Sep-2013; Online first: \\ 10-Sep-2013 \\ Copyright: (c) 2013 Bejiqi R. This is an open- \\ access article distributed under the terms of \\ the \\ which permits \\ wich permits unition, \\ aniginat aucth an source are credied. \\ are credited. \\ Competing Interests: The authors have \\ declared that no competing interests exist.
}

\begin{abstract}
Double-chambered left ventricle (DCLV) is a rare congenital anomaly, and only a few cases have been reported in the literature, in which a 2-chambered LV is separated by the interventricular septum or an abnormal muscle bundle.

We report a case of a girl who was presented at tertiary level for cardiological examination where, during the routine examination systolic murmur was registered. After echocardiographical examination DCLV was confirmed. Anomaly was associated with idiopathic hypertrophic cardiomyopathy $(\mathrm{HCM})$, severe mid cavity obstruction of left ventricle and high intra-ventricular peak pressure.
\end{abstract}

\section{Introduction}

Outpouching of the left ventricle is a rare condition with heterogeneous causes ranging from congenital abnormalities, such as diverticula or muscle bands, to complications secondary to myocardial infarction, such as aneurysm and pseudoaneurysm. Distinguishing among these etiologies is challenging but of great clinical importance given the wide range of risks and implications involved [1, 2].

Double chambered is a term that has been used to describe the subdivision of a ventricle because of anomalous septum or muscle bundle. Subdivision of the left ventricular cavity is a rare cardiac anomaly compared with subdivision of the right ventricle [2].

Hypertrophic cardiomyopathy is an inherited heart muscle disease with an estimated prevalence in the adult population of 1:500. Owing to the autosomal dominant nature of this disease, persons who have a parent with hypertrophic cardiomyopathy have a $50 \%$ risk of inheriting the mutation. From the genetics point of view, the disease is highly variable with respect to the specific gene mutation and degree of penetration. The most characteristic morphological abnormality in children with $\mathrm{HCM}$ is the excessive hypertrophy of the ventricle in absence of other cardiac or systemic diseases that could produce left ventricular hypertrophy [3]. Often, it is asymmetric in nature, with a preference for ventricular septum and occurs either in sporadic or familial forms. Often, children lack symptoms for a long period and frequently, the disease is detected due to the presence of a murmur or an arrhythmia. Classically, symptoms include pulmonary congestion, fatigue, palpitations, chest pain, syncope and congestive heart failure. A number 
of pathophysiological components and processes are identified: systolic dysfunction and left ventricular outflow tract obstruction, diastolic dysfunction, coronary artery abnormalities, leading to myocardial ischemia, mitral regurgitation, arrhythmias and sudden cardiac death. M-mode and 2-D echocardiography with color-flow Doppler evaluation are the primary screening tool for the evaluation of HCM [3].

An atypical form of $\mathrm{HCM}$ is presented with mid-cavitary obstruction of the left ventricle forming "two left chambers" and causing the intracavitary pressure. This form involves selective hypertrophy and obstruction at the mid-left ventricular level. In children this type of $\mathrm{HCM}$ is very rare and it is quite likely that there is a distinct anatomic subtype of hypertrophic cardiomyopathy, with still clearly unidentified etiology. In adults, these findings may represent effects of a long-standing hypertension with relatively small left ventricular cavities in some individuals [3-5].

\section{Case report}

A 28-month-old girl, from normal pregnancy and absolutely health parents, weighing $16.3 \mathrm{~kg}$, during the routine pediatric examination systolic murmur was noted and for cardiological examination at tertiary level was referred. The child's growth and development was completely normal. There was no sweating or fatigue during feeding or normal activities. Complete clinical and cardiological examination was obtained. An arterial blood gas was within normal limits. Clinical examination demonstrated: a quite precordium, normal first heart sound, short midsystolic murmur 2-3/6 degree on the apex and left sternal border, and single second heart sound. Electrocardiogram showed: normal sinus rhythm, left axis deviation, and biventricular hypertrophy. A chest radiogram revealed an enlargement cardiac silhouette with a narrow mediastinum. The pulmonary vasculature was normal in appearance, and there were no infiltrates seen. Echocardiography demonstrated: normal systemic and pulmonary vein connection, with small interatrial communication. There were normal atrio-ventricular and ventriculoarterial connections. Cross-sectional echocardiography from apical four-chamber view, during diastole demonstrated symmetric hypertrophy of the left ventricle, and subaortic obstruction together with interventricular septal hypertrophy and thickening. In addition, a 2-dimensional echocardiographic examination revealed 2 chambers divided by the muscular hypertrophic mass. The walls of the apical chamber and the septum were not hypokinetic. The hypertrophy was symmetric and severe, causing the cavity of the left ventricle in be anatomically divided into two separate parts. The apical part has been seen as separated from the outlet and inlet portion but clearly was presented that actively takes part in left ventricle contractility.
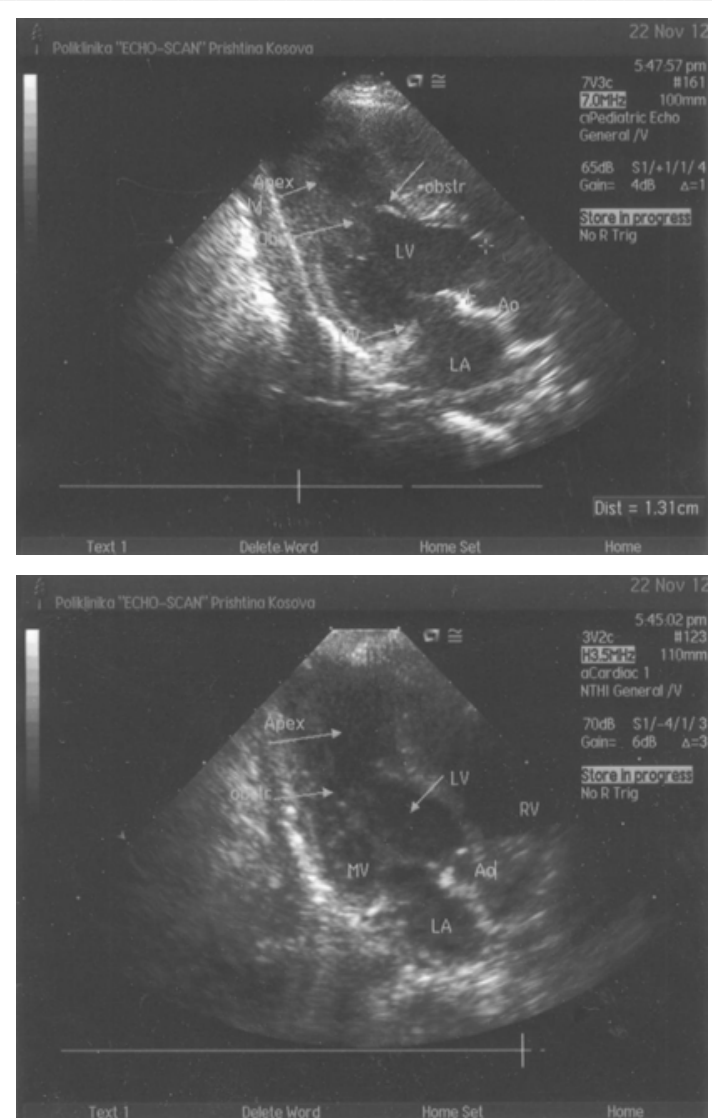

Figure 1: A four-chamber view of an echocardiogram showing a hypertrophic muscle mass originating from the left ventricular free wall and interventricular septum dividing it into 2 true chambers. $L V$ left ventricle, LA- left atrium, Ao - aorta, MV - mitral valve, RV-right ventricle.

By continuous Doppler waves, trivial mitral regurgitation was noted. A systolic gradient between the two left chambers was measured with the maximum velocity of $4.1 \mathrm{~m} / \mathrm{s}$ and gradient of $67 \mathrm{~mm}$ of mercury. The Color flow Doppler imaging showed in systole a narrow and turbulent color area on the middle part of left ventricle running to the mitral valve. This raised doubts that it is more likely that this is a case of double chamber left ventricle associated with $\mathrm{HCM}$ then a case with diverticulum or aneurysm of the left ventricle.

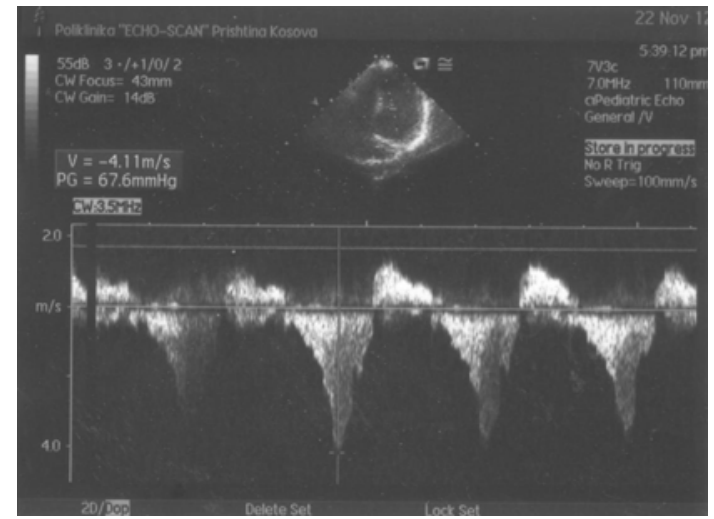

Figure 2: A four chamber view and continual Doppler shows high intracavitary pressure forming between 2 chambers. 


\section{Discussion}

The division of the ventricular chamber into two chambers characterizes double-chambered left ventricle by abnormal muscular tissue. It is best differentiated from left ventricular aneurysms and pseudo aneurysms by the fact that the doublechambered ventricle exhibits contractile motion during systole. Ventricular aneurysm lacks complete layering of the ventricular wall, and thus expands slightly due to the increased pressure during systole [1,2]. The differentiation between double-chambered left and right ventricles is clear as they have different pathophysiology. Double-chambered right ventricle (DCRV) is more common and often presents with murmur and exertional dyspnea. Studies have found that DCRV is associated with septal defects, tetralogy of Fallot, and transposition of the great arteries. Conversely, DCLV is commonly asymptomatic or associated with HCM. DCRV is often caused by a progressive thickening of the right ventricular septum due to the presence of anomalous muscle bundles. This causes a pressure gradient, and two chambers in series develop. In contrast, the chambers of a DCLV are in parallel and present less of a pressure gradient as both contracts synchronously. The DCLV etiology is less well known, but the anomaly is thought to be congenital and non-progressive [4-6].

Hypertrophic cardiomyopathy is now recognized as a genetic cardiac disease with an autosomal dominant pattern of inheritance, but with variable penetrance and sporadic phenotypical expression. There is genetic heterogeneity, with more than one gene being associated with the clinical manifestation and there is a great phenotypic variability, not only among unrelated families, but also within the same family. Over the recent years, knowledge about $\mathrm{HCM}$ has evolved enormously, mainly though advances in molecular genetics and in understanding of pathophisiological mechanisms, as well as our awareness of the great variability in its expression. When HCM is suspected, the prenatal detection is relatively easy after elimination of other cardiac diseases caused by the left ventricle hypertrophy, coronary anomalies, aortic valvular stenosis, aortic coarctation, etc.) $[4,5]$.

All forms of $\mathrm{HCM}$ have in common an inappropriate left ventricular hypertrophy, often with abnormal myofibril orientation and represent a diverse spectrum of disease with varying degrees of hypertrophic expression even among a given family. There is, however, a wide variation of expression within affected families. It is believed that the penetrance of the disease is incomplete during childhood and adolescence, increasing with age to nearly complete penetrance in adulthood. In general, there is no correlation between the severity of the disease and the symptomatology [3, 6]. Children often lack symptoms, and as a rule, HCM does not interfere with the physical development of the patient except in its severe forms. Affected children may remain asymptomatic for a long period of time. The clinical manifestation of the classic form of HCM results from systolic dysfunction and dynamic left ventricular outflow tract obstruction, diastolic dysfunction, mitral regurgitation, a high prevalence of arrhythmias and sudden cardiac death [7-9].

This rare congenital disorder is best classified as a 'double-chambered left ventricle', a term which has been used to describe the subdivision of an LV cavity by an abnormal septum or muscle bundle into two chambers. Only a few cases with variable morphologies have been reported in the literature, most with either a diverticular appearance or small contracting chambers attached to the LV lateral wall or within the apex [10-13]. Gerlis et al reported the largest series (3 cases), which consisted of patients who had endomyocardial fibroelastosis and cardiomyopathy. All of the patients died in the first year of life from the cardiomyopathy. The etiologic mechanism is unknown, and there is often an associated cardiomyopathy. However, there was no evidence of LV failure or cardiomyopathy in our case. Gerlis et al suggested that the endocardial fibroelastosis rendered the main chamber noncontractile and that the functional portion of the LV was derived from intratrabecular myocardial sinusoid expansion [14]. In our case, the etiology is likely to be related to cardiomyopathy despite that she has a normal ventricular function. Joy et al described a case of DCLV in which the patient had false tendons of fibrous or fibromuscular bands less than $3 \mathrm{~mm}$ in diameter crossing the LV cavity either between the LV free wall and the interventricular septum or between two papillary muscles [15]. Rickli et al reported a case of DCLV with a prominent fibromuscular ridge distal to the papillary muscles that divided the LV cavity into a small apical portion and a large basal portion. In the present DCLV case, a fibromuscular mass was seen to divide the LV into a small basal portion and a large apical portion. In our case, the associated septum was derived from the interventricular septum and the LV free wall, and it was in a floating formation that separated the LV cavity into 2 chambers. One of the reports described a patient with a large accessory chamber lying anterolateral to the main LV, with nonrestrictive communication occurring between the 2 chambers of the LV. In addition, the apical chamber was larger than the basal chamber [16].

Our case is unique in many aspects including: early age of diagnosis with normal growth, without other manifestations during the pregnancy, normal term delivery and asymptomatic neonatal and postnatal period.

\section{References}

1. Akdemir R, Balci MM, Ozer C, Karcaaltincaba M, Acikel S. Quadrichambered ventricles. Cardiol J. 2010;17:303-5.

2. Harvey Feigenbaum, William F. Armstrong, Thomas Ayan: Feigenbaum's Echocardiography, Lippincott Williams and 
Willkins, Philadelphia, 2005; 552-553.

3. Lakkis NM, Nagueh SF, Kleiman NS. Et al. Echocardiographyguided ethanos septal reduction for hypertrophic obstructive cardiomyopathy. Circulation. 1998; 98:1750-1755.

4. Ross J, Braunwald E, Gault JH et al. The mechanism of the intraventricular pressure gradient in idiopathic hypertrophic subarotic stenosis. Circulation. 1966; 34 (4) 558-578.

5. Shapiro LM, McKenna WJ. Distribution of left ventricular hypertrophy in hypertrophic cardiomyopathy: a two dimensional echocardiographic study. J Am Coll Cardiol. 1983; 2:437-444

6. Koz C, Yokusoglu M, Uzun M, Baysan O, Bulakbasi $N$. Double-chambered left ventricle with nonsustained ventricular tachycardia. Anadolu Kardiyol Derg. 2009;9:E5.

7. Ciro E, Marion BJ, Bonow RO, Cannon RO, Epstein SE Relation between marked changes in left ventricular outflow tract gradient and disease progression in hypertrophic cardiomyopathy. Am J Cardiol. 1984; 53:1103-1109.

8. Spirito $\mathrm{P}$, Maron BJ et al. Relation between extent of left ventricular hypertrophy and occurence of sudden cardiac death in hypertrophic cardiomyopathy J Am Coll Cardiol. 1990; 15:1521-1526.

9. 7. $\mathrm{H}$ Watkins, $\mathrm{H}$ Ashrafian, $\mathrm{W} \mathrm{J}$ McKenna, The genetics of hypertrophic cardiomyopathy: HEART. 2008;94(10): 1264 1269.

10. Breithardt OA, Ropers D, Seeliger T, Schmid A, Von Erffa J, Garlichs $C$, et al. A heart within the heart: double-chambered left ventricle. Eur J Echocardiogr. 2008;9:739-41.

11. Zhang XY, Cao TS, Yuan LJ. Double-chambered left ventricle in echocardiography. Echocardiography. 2012;29(3):E67-8.

12. Harikrishnan S, Sivasankaran S, Tharakan J. Double chambered left ventricle. Int J Cardiol. 2002;82:59-61.

13. Vaidyanathan $D$, Prabhakar $D$, Selvam $K$, Alegasan $R$, Thirunavukarasu N, Muthukumar D, Isolated congenital left ventricular diverticulum in adults. Indian Heart J. 2001;53:2113.

14. Gerlis LM, Patridge JB, Fiddler GI, Williams G, Scott O. Two chambered left ventricle: three new varieties. Br Heart J. 1981; 46:278-84.

15. Joy J, Subramanyan R, Sandhyamani A, Balakrishnan KG. Double chambered right ventricle with anomalous muscle bundles and false tendons in the left ventricle. Indian Heart $\mathrm{J}$. 1980;42:58-61.

16. Rickli $\mathrm{H}$, Attenhofer Jost $\mathrm{CH}$, Locher JT, Jenni RE. Doublechambered left ventricle: Unusual presentation with apical defect in a thallium scan in a middle-aged, asymptomatic woman. Echocardiography, 1999;16:387-91. 